\title{
Longterm Prognosis for Corneal Grafting in Herpes Simplex Keratitis
}

\author{
L. A. FICKER, C. M. KIRKNESS, N. S. C. RICE and A. D. McG. STEELE
}

London

\begin{abstract}
Summary
A previously reported cohort of patients ${ }^{4}$ was reviewed after mean follow-up of 10.9 years. The overall probability of survival was $45 \%$, but first grafts had a greater probability of survival than second $(P=0.12)$ or further $(P=0.19)$ grafts. Preoperative active keratitis adversely affected survival $(P=0.123)$. The major cause of failure was graft rejection. Regrafts were more likely to fail from rejection episodes $(P=0.0005)$. Antiviral prophylaxis improved the outcome for rejection $(P=0.005)$ and reduced the incidence of HSK recurrence complicating rejection. Suppurative keratitis occurred in $12.4 \%$ of grafts as a complication of epitheliopathy including HSK recurrence. The outcome in these cases was particularly poor. Loose continuous graft sutures resulted in graft failure in $10.6 \%$ of grafts which may be improved by using interrupted suturing. Our results suggest the longterm prognosis for grafting in herpes simplex keratitis are not as good as may have been predicted from previous analyses.
\end{abstract}

The purpose of this review was to establish the longterm survival of corneal grafts performed for Herpes Simplex Keratitis (HSK) and to determine the major factors which influence outcome. Outcome can be expressed by survival curves which allow statistical analysis of patients with unequal follow-up and different entry times into the study period. Previous studies have reported results for mean follow-up periods less than five years and it was our impression that survival curves derived from these data were likely to be incomplete, resulting in unrealistically high probabilities of survival.

Keratoplasty was introduced as a therapeutic strategy in the management of HSK prior to the availability of effective antiviral drugs. Diseased corneal tissue was excised and replaced by healthy cornea with the expectation of clinical improvement. ${ }^{1,2}$ Many of those early procedures were lamellar keratoplasties and were performed while the keratitis was active. A high incidence $(65 \%)$ of early stromal recurrence in lamellar grafts was reported by Rice and Jones in 1973. 3 Penetrating keratoplasty has since become the accepted procedure for HSK although the indications have changed following the introduction of effective topical antivirals. The present role of keratoplasty is mainly in visual rehabilitation.

When antivirals were administered topically as postoperative prophylaxis, epithelial toxicity was found to be a problem with the attendant complications of persistent epithelial defects and graft vascularisation. ${ }^{3}$ This practice was discontinued at Moorfields Eye Hospital without increasing the risk of HSK

From Cornea Clinic, Moorfields Eye Hospital, London.

Correspondence to: Linda Ficker FRCS, Dept of Clinical Ophthalmology, Institute of Ophthalmology, Moorfields Eye Hospital, City Road, London EC1.

Presented at the Annual Congress of the Ophthalmological Society of the United Kingdom, April, 1988. 
recurrence. A later report in $1980,{ }^{4}$ however, indicated there was an increased risk of HSK recurrence if graft rejection was treated with intensive topical steroids in the absence of antiviral prophylaxis. Treatment of rejection has since included antivirals, but their influence upon longterm graft survival has not been evaluated.

Risk factors for graft survival have been identified in previous studies, but the clinical features of HSK vary widely and the distribution of these varies between studies. There is not always concordance with respect to certain risk factors: Rice and Jones ${ }^{3}$ found the success rates for clear grafts to be the same for cases which were clinically active $(n=22)$ and inactive $(\mathrm{n}=28)$ at the time of keratoplasty. Cobo et $a l^{4}$, however, established a significant difference in the 2 year survival of grafts: $69 \%$ survival in 99 grafts in quiet eyes compared with $44 \%$ in 33 grafts performed in inflamed eyes. Cohen et $a l^{5}$ reported a series of 107 eyes grafted for HSK of which $93 \%$ were quiet at the time of surgery. Since such a small number were inflamed, it is difficult to draw conclusions from their finding that there was no difference in the outcome for quiet and inflamed eyes.

Other risk factors implicated as threats to graft survival are preoperative corneal vascularisation, size of the donor button, regrafting, repeated rejection episodes, HSK recurrence and adjunctive surgery. We have evaluated these risk factors within the constraints of the database available from our review and we have considered additional factors such as suturing and the efficacy of antiviral prophylaxis for rejection episodes.

\section{Patients and Methods}

We reviewed 65 (71\%) of the 91 patients who underwent penetrating keratoplasty for HSK at Moorfields Eye Hospital between 1967 and 1978. This was the cohort previously reported in $1980^{4}$. The major indication for grafting was corneal opacity involving the visual axis. Grafts performed for other reasons included active HSK $(\mathrm{n}=11)$ and supervening suppurative keratitis $(n=5)$; nine of these 16 hot eyes were perforated at the time of surgery. Ten patients had previously undergone grafts: 6 lamellar and 4 penetrating.
Since 1967 a total of 96 grafts have been performed in these 66 eyes. Hence the series included 62 first grafts and 34 regrafts. Follow-up ranged between 12 months and over 20 years for 66 eyes of 65 patients $(93 \%$ were followed more than 5 years; mean 10.9 years, median 10.3 years).

Donor tissue was obtained from the Moorfields Eye Bank and prior to 1979 only moistchamber stored whole eyes were available. The material was refrigerated and used within a maximum 48 hours of enucleation and mostly within 24 hours. Penetrating grafts ranging between $5 \mathrm{~mm}$ and $9 \mathrm{~mm}$ diameter $(84 \%$ between $7 \mathrm{~mm}$ and $8 \mathrm{~mm})$ were sutured using continuous $10 / 0$ monofilament nylon. Twelve patients underwent cataract extraction (11/12 intracapsular) and 5 underwent vitrectomy at the time of keratoplasty. Steroids were administered postoperatively and gradually tailed off over a 12 month period according to clinical signs.

Corneal rejection was diagnosed in the presence of anterior chamber inflammation with keratic precipitates (KP) limited to the donor cornea. Rejection episodes were treated with intensive topical steroids and, during the last 20 years, antiviral prophylaxis was introduced. One of three antivirals was prescribed over that period: Idoxuridine, Trifluorothymidine or Acyclovir (Acyclovir was only available after 1979).

Epithelial HSK recurrence was treated with topical antivirals. Additional topical steroids were used if recurrence was associated with anterior uveitis. Stromal recurrence was diagnosed in the presence of stromal infiltrates and oedema with a subjacent endothelial ring of keratic precipitates. Stromal recurrence was treated with both topical antiviral and steroid. Loose sutures were managed in several ways: repeated trimmings, total removal or the application of a therapeutic bandage lens. Two grafts were resutured and one was replaced.

Since follow-up periods varied between grafts in this series and many grafts remained clear when last examined, the data obtained are censored and were therefore subjected to appropriate statistical analysis. Survival curves were derived according to the method of Kaplan and Meier. ${ }^{6}$ The influence of fac- 
tors including regrafting, suturing, rejection and recurrent HSK upon graft survival was evaluated. Graft failure was defined as irreversible loss of graft clarity.

\section{Results}

At final follow-up 55\% of patients had a clear graft (Fig. 1) and $47 \%$ of the patients had corrected visual acuities of $6 / 12$ or better. Nine patients were found to have poor vision for reasons other than graft failure: two had cataracts, two had retinal detachments, one suffered a traumatic expulsive haemorrhage, two had aphakic glaucoma and two were amblyopic.

The overall survival curve (Fig. 2) for the 96 grafts stabilised at 6 years when the probability of survival was $50 \%$. The final probability of survival at 216 months was $45 \%$. When the results were analysed separately for first grafts, regrafts and subsequent grafts, the probabilities for survival at 5 years were $54 \%$ for first grafts, $39 \%$ for second grafts and $30 \%$ for subsequent grafts (Fig. 3). The 10 year survival was $54 \%$ for first grafts and $30 \%$ for second grafts. While the probability of survival of first grafts stabilised at 3 years, the probability of survival of second grafts continued to fall for at least six years. The maximum follow-up for third or additional grafts was 65 months at which time the rate of failure was greater than for first grafts or second grafts. Statistically, first grafts had a better prognosis for survival than second grafts $(\mathrm{P}=0.12)$ or subsequent grafts $(\mathrm{P}=$ $0.19)$.

Since corneal vascularisation has been implicated as a major determinant of graft

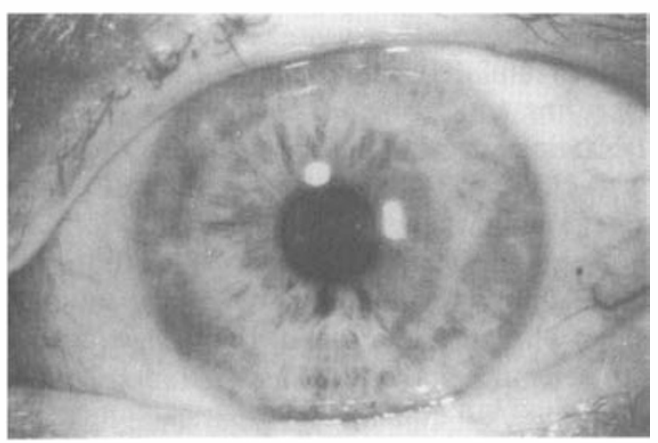

Fig. 1. A clear graft 12 years after surgery for herpes simplex keratitis. survival, survival curves for first grafts were derived for three subgroups defined as nonvascularised, partially vascularised (1-3 quadrants) and totally vascularised (Fig. 4) according to criteria adopted in previous studes. ${ }^{4.7}$

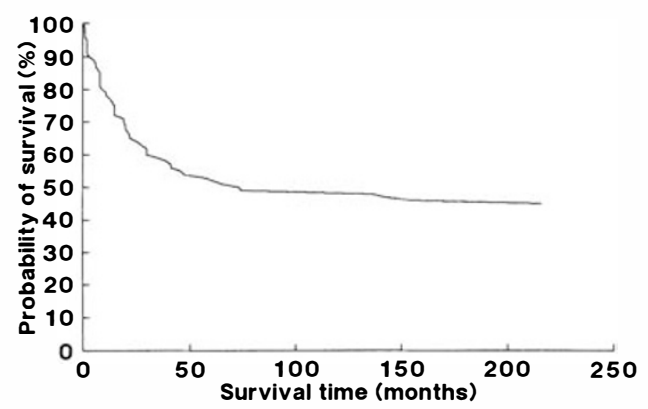

Fig. 2. The Kaplan-Meier survival curve, for all grafts in the series.

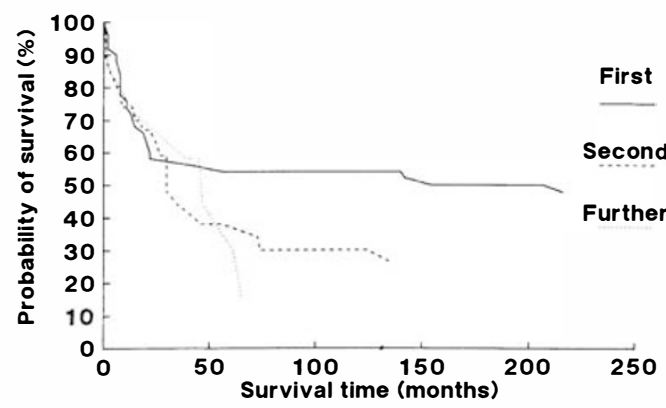

Fig. 3. The survival curves for grafts segregated according to whether the graft was the first, second or further graft in that eye.

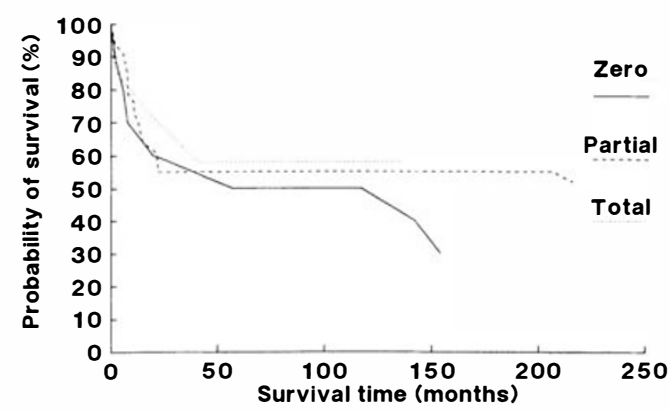

Fig. 4. The survival curves for grafts segregated according to the degree of pre-operative vascularisation. Partially vascularised implies that there was deep or superficial vascularisation in 1-3 quadrants of the cornea. Total vascularisation implies that all quadrants were involved. 
The survival curves for these subgroups did not demonstrate a tendency for totally vascularised corneae to have a significantly worse prognosis than non-vascularised corneae, as determined by Gehan analysis. Corneal vascularisation was as follows for first grafts: $17 \%$ zero, $69 \%$ partial and 13\% total, while for regrafts the distribution shifted: $0 \%$ zero, $39 \%$ partial and $61 \%$ total. A subgroup of 5 patients had undergone lamellar grafts prior to recruitment for penetrating keratoplasty. Four of these were totally vascularised, but the probability of survival for the subgroup was found to be $60 \%$ which compares with that for first grafts and does not appear adversely affected by corneal vascularisation (Fig. 5).

Sixteen eyes were inflamed at the time of keratoplasty and the probability of survival for this subgroup stabilised at 46 months when it was $40 \%$, with a final probability of $28 \%$ at 154 months (Fig. 6). Survival of quiet eyes was $50 \%$ which was significantly greater $(\mathrm{P}=0.13)$.

The survival of 11 grafts performed in combination with intracapsular cataract extraction was poor, falling steadily over a period of 74 months to $10 \%$. This group, however, included 6 regrafts and 3 eyes which were inflamed when operated upon.

Primary failure occurred in 4 grafts: one inflamed eye and 3 quiet eyes, including both eyes of one patient with bilateral disease. Graft rejection (Fig. 7) was an important cause of failure. Of the 58 failures, rejection was either the immediate cause or resulted in irreversible complications in 29 cases. The incidence of graft rejection was $62 \%$ for first grafts and $55 \%$ for regrafts. The mean postoperative interval for the occurrence of graft rejection was 19 months for both groups. Fourteen grafts, including 3 regrafts, suffered multiple rejection episodes. Of these, seven failed and seven survived. Hence $25 \%$ of failures from rejection were associated with between two and six rejection episodes.

The failure rate from rejection was, however, greater for regrafts $(68 \%)$ than for first grafts $(39 \%)$. This is a significant difference $(\mathrm{P}=0.0005)$. Rejection episodes were treated with intensive topical steroids, but without antiviral prophylaxis in $58 \%$ of affected first grafts and $50 \%$ of regrafts. When antiviral prophylaxis was included in the regime for graft rejection, the failure rate was significantly reduced, from $54 \%$ to $17 \%$, for first episodes of rejection $(\mathrm{P}=0.005)$. The incidence of HSK recurrence was reduced from $35 \%$ to $4 \%$ with the addition of

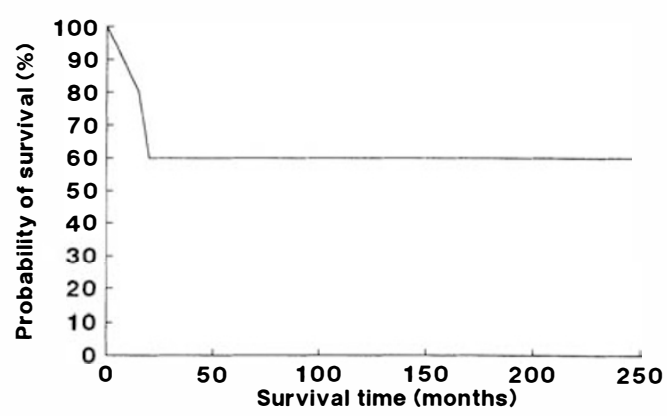

Fig. 5. The survival curve for those eyes having a penetrating keratoplasty where there had been a pre-existing lamellar graft.

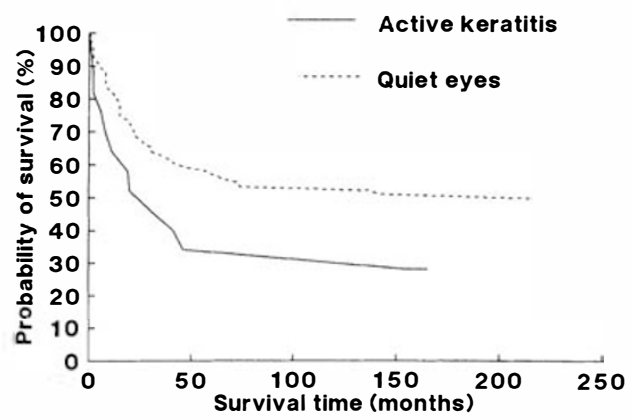

Fig. 6. The survival curve for grafts segregated according to the inflammatory status of the eye at the time of keratoplasty.

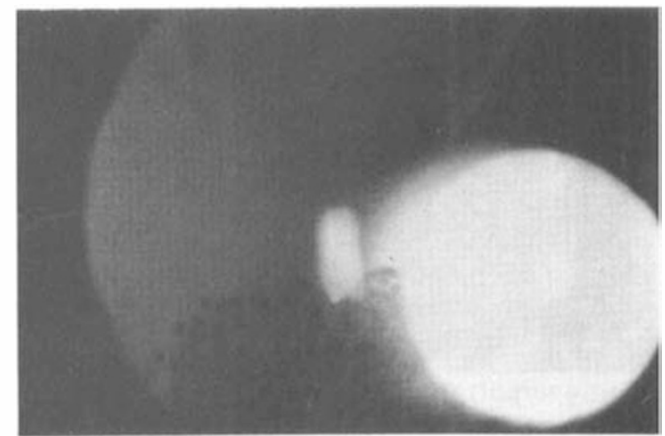

Fig. 7. High magnification slit lamp photograph showing a graft with rejection (retro-illumination). 
antiviral prophylaxis. Thus a modification in the treatment regime introduced a favourable bias for survival of regrafts since these were performed later in the study.

Rejection episodes were complicated by raised intraocular pressure, up to $48 \mathrm{~mm} \mathrm{Hg}$, in 10 patients of whom 4 had regrafts. The majority $(9 / 10)$ were managed medically and intraocular pressure returned to normal with the resolution of the rejection episode. Glaucoma complicated the postoperative course of a further 6 patients of whom two required trabeculectomy.

HSK recurrence occurred in $41 \%$ of the series and was predominantly epithelial (90\%) (Fig. 8). Early recurrence (within 12 months of keratoplasty) was usually associated with an event such as rejection or suture removal. Twelve cases complicated a rejection episode treated without antiviral

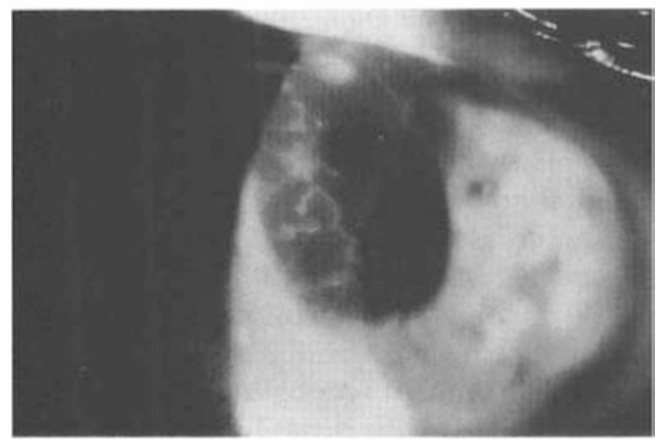

Fig. 8. Slit lamp photograph showing recurrent epithelial herpetic disease, the ulcer just spans the graft-host interface.

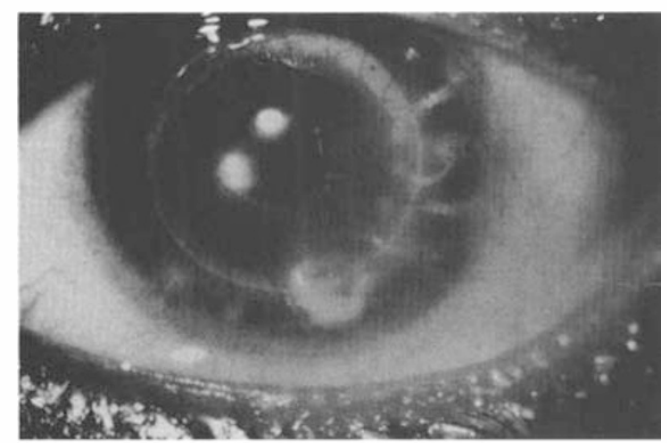

Fig. 9. The running suture has broken and becomes loose, attracting mucus and vascularisation. If left untreated rejection or epithelial recurrence may be triggered. prophylaxis (mean postoperative delay of 11.6 months); eight of the 12 failed. In seven cases, epithelial recurrence followed suture removal, loosening of the graft suture or early glaucoma surgery (mean postoperative delay of four months). The remaining 16 cases of epithelial recurrence occurred at a mean postoperative delay of 28 months. When epithelial recurrence occurred alone, only one graft subsequently failed due to perforation. Four grafts, however, developed disciform keratitis (mean postoperative delay of 36 months) and three of the four resulted in graft failure.

The role of sutures in graft survival was evaluated. Elective removal was performed in 48 cases and in only four were complications precipitated: two rejection episodes and two epithelial HSK. Of these, two grafts failed $(4.2 \%)$. Loose sutures (Fig. 9) were observed in 36 cases and managed as follows: piecemeal removal in 20 , complete removal in eleven and application of a therapeutic bandage lens with subsequent removal in five. Of these 36 patients, 14 had associated rejection episodes of which six led to failure. A further 4 grafts failed in association with loose sutures: one suppurative keratitis, two vascularised grafts and one unstable graft which was regrafted. Thus 10 failures resulted from complications of loose graft sutures (10.6\% of all keratoplasties).

Suppurative keratitis compounded complications in 12 grafts. The predisposing events were as follows: epithelial HSK recurrence $(n=5)$, ocular surface disorders including band keratopathy and sequelae of vernal keratoconjunctivitis $(n=6)$ and loose graft sutures $(n=1)$. The pathogens involved were Staphylococci $\quad(n=5), \quad$ Streptococcus pneumoniae $(n=4)$, Moraxella, ${ }^{1}$ Neisseria cattarhalis $^{1}$ and a Gram negative bacillus. ${ }^{1}$ No eye was lost due to infection, but three perforations required immediate regrafting and seven other grafts were scarred and were electively replaced.

\section{Discussion}

Survival curves provide a means of expressing results for a group of patients who are followed for different periods and for whom the results of treatment vary with time. The 
Kaplan Meier curves are recognised statistical expressions ${ }^{6}$ and have been applied to the results for grafting in HSK by previous authors. ${ }^{4,8}$ Survival curves were used to evaluate differences between first grafts and regrafts. They were also used to determine the influence of possible risk factors such as preoperative inflammation and corneal vascularisation.

The survival of first, second and subsequent grafts initially fell in parallel. The 2 year survival of first grafts in this series was $58 \%$ compared with $66 \%$ for the original cohort reported by Cobo. ${ }^{4}$ At 2 years, the $58 \%$ survival of first grafts compared with $67 \%$ for second grafts and $72 \%$ for subsequent grafts, but these differences are not significant. The major separation of survival curves occurred after 4 years at which time the curves were stabilising for first and second grafts.

Our study revealed a continuing change in the pattern of survival beyond the mean follow-up of about 4 years for previous reports. ${ }^{3-5,7,9,10}$ Graft survival has not stabilised at 4 years, hence the longterm outcome has not hitherto been established. Statements about longterm survival are probably best made when survival curves are seen to be stabilising. The difficulties of comparing results from different series with varying follow-up are compounded by the variation between studies with respect to clinical features, management and surgical techniques as discussed by Fine. ${ }^{9}$

Graft survival has been correlated with preoperative corneal vascularisation. ${ }^{4}$. This series demonstrated that regrafts were more frequently vascularised preoperatively, thus vascularisation may not be an independent variable. To test this we considered the results for three subgroups of first grafts (the largest group, with the longest follow-up) and the small group which previously had lamellar grafts and were highly vascularised. These did not demonstrate a significant difference in survival on the basis of corneal vascularisation which we do not therefore consider to be a major risk factor. Cohen et $a l^{5}$ have also reported this conclusion. Vascularisation theoretically threatens the immunological privilege of the cornea, ${ }^{11}$ but this is probably countered by steroid administration.

The longterm survival of grafts performed during active HSK was poor $(30 \%)$ but the 4 year survival was $60 \%$. Cobo previously reported $^{4}$ a difference between the 2 year survivals of inflamed eyes $(44 \%)$ and quiet eyes $(69 \%)$. The failure rate can therefore be expected to vary considerably with time. This would account at least in part for the $77.3 \% 2$ year survival reported by Rice and Jones ( 22 of 50 eyes were inflamed $)^{3}$ as compared with the $15 \%$ survival at 4.3 years reported by Foster $^{7}$ for a series in which 53 of 82 eyes were inflamed. These authors did not use actuarial techniques for survival analysis. Polack and Kaufman ${ }^{10}$ found only $45 \%$ of 20 grafts for active HSK remained clear after 12 months, but steroids were not routinely administered postoperatively. The causes of failure in these cases include technical difficulties and early damage of donor endothelium during severe anterior segment inflammation, as discussed by Cobo et al. ${ }^{4}$

There was overlap of inflamed eyes and those which underwent adjunctive surgery, in the series we report. It is therefore difficult to implicate the additional surgery as a distinct risk factor. It reflects more severe ocular disease resulting from kerato-uveitis and this more likely explains the association between cataract surgery and poor prognosis for grafting. Epstein et al ${ }^{12}$ found cataract $(\mathrm{P}=0.01)$ and glaucoma $(\mathrm{P}=0.003)$ to be risk factors for first grafts in HSK. The glaucomas were analysed and found to be related to inflammation and synechiae. Thus inflamed eyes appear to be associated with a need for adjunctive surgery and with complications including glaucoma and graft failure. Since most $(84 \%)$ donor buttons were $7-8 \mathrm{~mm}$ in diameter, we are unable to comment on the additional risk from large grafts.

We found the major cause of failure to be rejection. The criteria for rejection have been discussed in the past ${ }^{3,13}$ and differences in the criteria applied would account for the variable incidence reported in the literature. ${ }^{3-5,7-10,12}$ We consider anterior segment inflammation with donor keratic precipitates to indicate rejection. Epstein et al ${ }^{12}$ classify this as probable rejection and did not include 
these cases when evaluating incidence of rejection. We do not limit the diagnosis of rejection to those patients with an endothelial rejection line which we believe represents a later stage in the process. Early diagnosis of rejection allows prompt treatment of this destructive process. We acknowledge the difficulties of differentiating between graft rejection and HSK recurrence with secondary uveitis. $^{3,9}$ The higher incidence of rejection episodes reported in this as compared with some other series reflects our inclination towards early diagnosis, prior to the appearance of an endothelial line. An endothelial line was, however, present in $55 \%$ of rejection episodes diagnosed.

Rice and Jones reported the incidence of graft oedema with uveitis to be $57.9 \%$ which compares with our incidence of rejection: $62 \%$ for first grafts and $55 \%$ for second grafts. Epstein et al ${ }^{12}$ diagnosed definite rejection in the presence of an endothelial or epithelial rejection line or subepithelial infiltrates confined to the donor and not associated with an acute conjunctivitis. ${ }^{13}$ They found the incidence of rejection to be $16.6 \%$ for the first grafts and $23.7 \%$ for second grafts. This probably underestimates the true incidence, as they acknowledge, and this must be emphasised in the interests of maximising graft survival by aggressive treatment of rejection.

Treatment of rejection in our clinic now includes antiviral prophylaxis. This has improved the outcome for rejection and reduced the incidence of epithelial disease which introduces the further threat of suppurative keratitis. It covers those cases in which HSK recurrence may be the primary event and others in which steroid-induced viral replication may occur. The precise pathophysiology remains unexplained, but the benefits of antiviral cover have been reported by many authors..$^{5,14,15} \mathrm{We}$ found the incidence of rejection to be the same for first grafts and regrafts, but the failure rate was higher for regrafts. The regrafts were not subject to a higher incidence of multiple episodes, nor were multiple episodes more likely to fail. Epstein et al, ${ }^{12}$ however, noted an increased incidence of rejection for regrafts and found multiple episodes to have decreased survival in HSK patients. The number of patients is small and the outcome for each episode will depend in part upon how promptly the patients presented for treatment. The role of HLA-DR antigen expression, ${ }^{16}$ particularly where a previous graft may have sensitised lymphocytes to similar or identical antigens, may also be pertinent. The small statistical database is unlikely to allow definition of these various effects.

The incidence of HSK recurrence was $41 \%$ and this compares with the $47 \%$ reported by Fine $^{9}$ for 4 year follow-up. HSK recurrence was more frequently epithelial $(90 \%)$ than stromal; Fine observed $72.7 \%$ of recurrences were epithelial. We identified trigger events such as loosening of a continuous suture or surgical manipulation. In the absence of such events we found epithelial recurrence to be unusual ( $4 \%$ of all grafts) within 12 months of surgery, as did Fine. ${ }^{9}$ We do not therefore believe postoperative antiviral prophylaxis is justified since it is known to have disadvantages due to toxicity ${ }^{3}$ and delayed wound healing. ${ }^{14,17}$ The recurrence rate reported at 2 years by $\mathrm{Cobo}^{4}$ was $18 \%$ for cases managed without postoperative antivirals and $15 \%$ for cases managed with antivirals, ${ }^{14}$ but the latter had a $25 \%$ incidence of persistent epithelial defects.

Epithelial recurrence was complicated by suppurative keratitis in 5 cases of which two perforated, two were regrafted and one caused severe corneal scarring. Secondary infection should be excluded early in indolent cases of HSK recurrence which do not respond to antiviral treatment, since the outcome for bacterial superinfection tends to be particularly poor.

Stromal recurrence was less common, but the failure rate was high. It is likely that some early cases of stromal recurrence may be interpreted as rejection episodes. Although the management includes steroid and antiviral in both cases, differences in diagnosis will influence the notional causes of failure.

Suturing technique has not been accorded attention in past reports yet we identified serious complications related to loose sutures in $10.6 \%$ of our grafts. HSK recurrence and rejection were both triggered by loose 
sutures early in the postoperative period and these complications persisted or recurred when the continuous suture was trimmed repeatedly. This suggests that interrupted sutures (Fig. 10) would be preferable in the surgical management of HSK, both active and inactive at the time of keratoplasty, to allow removal of loose sutures without compromising graft stability. Since HSK results in uneven stromal thinning, these grafts are especially vulnerable to astigmatism and interrupted sutures offer the additional advantage of refractive manipulation.

The determinants of graft survival for HSK are multifactorial. Longterm survival stabilises at about 6 years, this process being more gradual for regrafts. Previous keratoplasty is an important preoperative risk factor. This means careful patient selection for regrafting with respect to other risk factors, compliance with treatment and visual needs. Suppression of preoperative inflammation and the early recognition of postoperative rejection are important. The major cause of graft failure is rejection, especially when complicated by HSK recurrence. Epithelial HSK recurrence is frequently complicated by suppurative keratitis which should be excluded by corneal scrapes if infiltrates are observed. Epithelial toxicity of antivirals contributes to this risk and should be monitored, since a change of antiviral may reduce complications. Finally, there is a strong case for interrupted sutures for both inflamed and quiet eyes. Attention to all these factors is likely to improve longterm survival. Major improvements in the future will probably depend upon new means of immunomodulation.

\section{References}

${ }^{1}$ Ormsby HL: Keratoplasty for herpetic keratitis. Am J Ophthalmol 1958, 45: 179.

${ }^{2}$ Fine M: Treatment of herpetic keratitis by corneal transplantation. Am J Ophthalmol 1958, 46: 671.

${ }^{3}$ Rice NSC and Jones BR: Problems of corneal grafting in herpetic keratitis. CIBA Foundation Symposium 15: Corneal graft failure. Ed Jones BR. Amsterdam: Elsevier 1973, 22139.

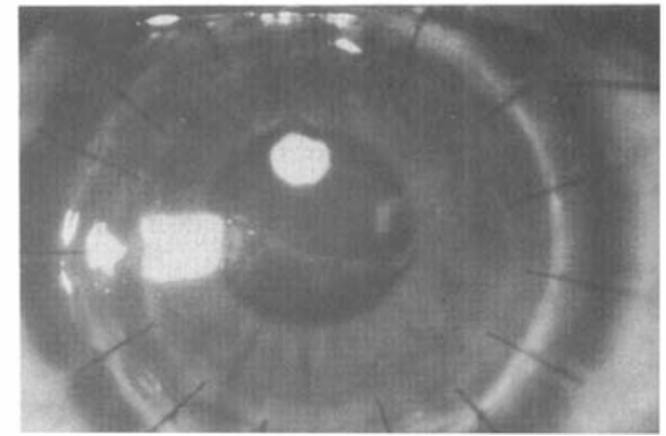

Fig. 10. A graft illustrating the recommended technique of interrupted suturing. This graft is about 3 weeks post operative and still shows a little epithelial disturbance.

${ }^{4}$ Cobo LM, Coster DJ, Rice NSC, Jones BR: Prognosis and management of corneal transplantation for herpetic keratitis. Arch Ophthalmol 1980; 98: 1755-9.

${ }^{5}$ Cohen EJ, Laibson PR, Arentsen JJ: Corneal transplantation for herpes simplex keratitis. Am J Ophthalmol 1983, 95: 645-50.

${ }^{6}$ Kaplan EL and Meier P: Nonparametric estimation from incomplete observations. $\mathrm{Am} \mathrm{J}$ Stat Assoc 1958, 53: 457-81.

${ }^{7}$ Foster CS and Duncan J: Penetrating keratoplasty for herpes simplex keratitis. $\mathrm{Am} \mathrm{J}$ Ophthalmol 1981; 92: 336-43.

${ }^{8}$ Volker-Dieben HJ, Kok-van Alphen CC, D'Amaro J, de Lange P. The effect of prospective HLA-A and $B$ matching in 288 penetrating keratoplasties for herpes simplex keratitis. Acta Ophthalmol 1984, 62: 513-23.

${ }^{9}$ Fine $\mathrm{M}$ and Cignetti FE: Penetrating keratoplasty in herpes simplex keratitis. Arch Ophthalmol 1977, 95: 613-6.

${ }^{10}$ Polack FM and Kaufman HE: Penetrating keratoplasty in herpetic keratitis. Am $J$ Ophthalmol 1972, 73: 908-13.

${ }^{11}$ Khoudadost AA and Silverstein SB: Studies on the nature of the privilege enjoyed by corneal allografts. Invest Ophthalmol 1972, 11: 13748.

12 Epstein RJ, Seedor JA, Dreizen NG, Stulting RD, Waring GO, Wilson LA, Cavanagh HD: Penetrating keratoplasty for Herpes simplex keratitis and keratoconus. Ophthalmol 1987, 94: $935-44$.

${ }^{13}$ Krachmer JH and Alldredge DC: Subepithelial infiltrates - a probable sign of corneal transplant rejection. Arch Ophthalmol 1978, 96: 2234. 
${ }^{14}$ Langston RHS, Pavan-Langston D, Dohlman $\mathrm{CH}$ : Antiviral medication and corneal wound healing. Arch Ophthalmol 1974, 92: 509-13.

${ }^{15}$ Patterson A and Jones BR: The management of ocular herpes. Trans Ophthalmol Soc UK 1967, 87: 537-79.

${ }^{16}$ Young E, Stark WJ, Prendergast RA:
Immunology of corneal allograft rejection HLA-DR antigens on human corneal cells. Invest Ophthalmol Vis Sci 1985, 26: 571-4.

17 Puelhorn G, Sosath G, Thiel H-J: The effect of 5-iodo-2-deoxyuridine (IDU) and dexamethasone on corneal wound healing in the rabbit. Acta Ophthalmol 1978, 56: 40-52. 